\title{
Distributed Asynchronous Fusion Algorithm for Sensor Networks with Packet Losses
}

\author{
Tianpeng Chu, Guoqing Qi, Yinya Li, and Andong Sheng \\ School of Automation, Nanjing University of Science and Technology, Nanjing 210094, China \\ Correspondence should be addressed to Tianpeng Chu; chutianpeng@gmail.com
}

Received 20 September 2013; Revised 3 December 2013; Accepted 4 December 2013; Published 9 January 2014

Academic Editor: Lifeng Ma

Copyright (C) 2014 Tianpeng Chu et al. This is an open access article distributed under the Creative Commons Attribution License, which permits unrestricted use, distribution, and reproduction in any medium, provided the original work is properly cited.

This paper is concerned with the problem of distributed estimation fusion over peer-to-peer asynchronous sensor networks with random packet dropouts. A distributed asynchronous fusion algorithm is proposed via the covariance intersection method. First, local estimator is developed in an optimal batch fashion by constructing augmented measurement equations. Then the fusion estimator is designed to fuse local estimates in the neighborhood. Both local estimator and fusion estimator are developed by taking into account the random packet losses. The presented estimation method improves local estimates and reduces the estimate disagreement. Simulation results validate the effectiveness of the proposed distributed asynchronous fusion algorithm.

\section{Introduction}

Distributed fusion and estimation problem over peer-topeer sensor networks has attracted significant interest in the research community, because of its variety of applications, such as environmental monitoring, surveillance, and target tracking, [1-10]. In these applications, every sensor in the network does not only take measurements in a parallel manner but also acquires information from neighbors and processes it to get an estimate. Compared with the centralized fusion fashion, the main advantage of distributed fusion estimation is the computation burden alleviation and robustness enhancement.

In general, two main approaches are presented in the literature to solve the distributed fusion and estimation problem. The first approach is the consensus approach. The consensus approach was proposed in $[11,12]$, where local measurements are exchanged among neighbors to get local estimates, and then by using average consensus algorithms among neighbors every sensor in the network gets the same estimate in steadystate. In order to obtain the same estimate at every sensor in the network, the consensus approach may iterate several times for each new measurement. This is highly undesired when estimating the state of dynamic systems where new measurements need to be processed in a timely manner. The second approach is the diffusion approach. The diffusion approach was presented in [13], in which the estimates of local filters are calculated individually at each sensor by using the data from the neighborhood, and then the local estimates from the neighborhood are fused locally by a convex combination. Therefore, the diffusion approach is well suited for estimating the state of dynamic systems where new measurements are being taken in real time. Though the adaptive weights for the diffusion algorithm were presented in [14], the estimation error covariance information is not taken into account. In $[15,16]$, the covariance intersection (CI) algorithm was proposed, in which the coefficients of convex combination of the estimates are chosen by considering the error covariance information. Therefore, the CI algorithm was used to design the adaptive weights for the diffusion algorithm in [17]. Though the aforementioned works solved the distributed fusion and estimation problem in their specific perspectives, they did not take into consideration the packet dropouts which are usually unavoidable in networks.

Many results on control and state estimation in networked systems context are already presented for the problem of both unreliable communication links and measurement uncertainties. The interested readers may refer to [18-23] 
and the references therein for further information. We will review only those works that are closely related to the state estimation in sensor networks with packet dropouts.

The problem of packet dropouts which are usually unavoidable in sensor networks has gained a lot of attention [24-27]. Schenato addressed a minimum error covariance estimator for sensor networks with random packets losses in [28]. The critical data arrival rate which ensures the stability of Kalman filter is investigated in [29]. An optimal filter is proposed based on the probability of the packet dropouts in [30]. Sun proposed optimal linear estimators in the sense of minimum variance with respect to the packet dropout probability in [31]. Ma and Sun proposed a centralized linear optimal fusion estimator in the minimum variance sense in [32]. Though the aforementioned literatures deal with the random packet losses problem, the sensor asynchronous problem is not taken into account.

Most of the aforementioned works are developed under the synchronous assumption. And the real-world systems may usually be asynchronous. In regard to asynchronous sensor fusion, a series of linear weighted fusion (LWF) algorithms for two and more than two asynchronous sensors with and without feedback had been proposed separately in [3336]. By establishing state-space models at each sampling rate, a new fusion algorithm for asynchronous sensors had been presented in [37]. A minimum mean-squared error (MMSE) centralized asynchronous fusion algorithm had been presented in [38], which is suitable for arbitrary sensor number, sensor sampling rates, sensor initial sampling time instants, and fusion periods. By reconstructing the optimal centralized asynchronous fusion algorithm in [38], a so-called distributed asynchronous fusion algorithm is proposed in [39], which performs equivalently to the optimal centralized asynchronous fusion algorithm. Though the aforementioned works deal with the asynchronous sensor fusion problem, the random packet dropouts problem is not taken into account. Moreover, these works always need a fusion center to accomplish the estimation task.

Motivated by the above discussion, we consider the distributed estimation fusion problem for an asynchronous sensor network with random packet dropouts. The sensor network is considered to be a peer-to-peer network with no fusion center, and the communication channels of the sensor network are unreliable. The main contribution of our paper is the development of the distributed asynchronous fusion algorithm in a peer-to-peer sensor network with random packet dropouts. Our algorithm is developed as follows. Firstly, a Bernoulli random variable is employed to describe the random packet dropouts. Then, by constructing a local augmented measurement with the random packet dropouts under consideration, an asynchronous local estimator is obtained at each sensor to compute local estimate. Further, the fusion estimators at different sensors are presented by utilizing a weighted fusion criterion based on covariance intersection method, and the error covariance information of local estimates is used to compute the fusion weights. The main advantage of the proposed distributed asynchronous fusion algorithm is the practicality when dealing with a practical asynchronous sensor network with unreliable communication links.

The rest of the paper is organized as follows. In Section 2, the asynchronous distributed estimation problem is formulated. Then, design procedures for the asynchronous distributed fusion algorithm are presented in Section 3. The effectiveness of the proposed algorithm is demonstrated by a simulation example in Section 4. Finally, the conclusion is provided in Section 5.

\section{Problem Formulation}

Consider a continuous-time dynamic system described by the following linear stochastic differential equation:

$$
\dot{x}(t)=A(t) x(t)+G(t) w(t),
$$

where $x(t) \in \mathscr{R}^{d_{x}}$ and $w(t) \in \mathscr{R}^{d_{x}}$ is zero-mean white Gaussian noise with covariance matrix $q(t)$. Suppose that $\Phi\left(t_{k}, t_{k-1}\right)$ is the corresponding state transition matrix of $A(t), t_{k-1}$ is the time when the fusion center executed the last fusion operation or the initial time if $k=1, t_{k}$ is the oncoming fusion time instant, and $T=t_{k}-t_{k-1}$ is the fusion interval. Then, by discretizing the continuous-time linear system (1), we have

$$
x\left(t_{k}\right)=\Phi\left(t_{k}, t_{k-1}\right) x\left(t_{k-1}\right)+w\left(t_{k}, t_{k-1}\right),
$$

where $w\left(t_{k}, t_{k-1}\right)=\int_{t_{k-1}}^{t_{k}} \Phi\left(t_{k}, v\right) G(\tau) w(v) d v$ is zero-mean white Gaussian noise with covariance matrix $Q\left(t_{k}, t_{k-1}\right)=$ $\int_{t_{k-1}}^{t_{k}} \Phi\left(t_{k}, \tau\right) G(\tau) q(\tau) G^{T}(\tau) \Phi^{T}\left(t_{k}, \tau\right) d \tau$.

Observations of system (1) are obtained by a sensor network which deployed spatially. The sensor network which consists of $N$ distributed sensors is considered to be a peerto-peer network. The interconnection topology of the sensor network is denoted by $\mathscr{A}=\left\{L^{i, j}\right\} . L^{i, j}=1$ indicates that sensor $i$ and sensor $j$ are connected, otherwise $L^{i, j}=0$ implies that sensor $i$ and sensor $j$ are not connected. No fusion center exists in the sensor network, and every sensor acts also as an estimator which collects information only from its neighbors to generate local estimates. The set of sensors connected to the certain sensor $i$ is called the neighborhood of sensor $i$ and it is denoted by $\mathcal{N}_{i}$. Without loss of generality, assume that $i \in \mathcal{N}_{i}$ and the number of neighbors of sensor is given by the number of elements of $\mathcal{N}_{i}$, written as $\mathscr{D}_{i}$.

Suppose that the number of measurements provided by sensor $i$ during the $k$ th time interval is $n_{k}^{i}$. So observations during the $k$ th time interval of system (1) can be modeled as

$$
y_{k}^{i, j}=h_{k}^{i, j} x\left(t_{k}^{i, j}\right)+v_{k}^{i, j}, \quad i=1, \ldots, N ; j=1, \ldots, n_{k}^{i},
$$

where $h_{k}^{i, j}$ and $v_{k}^{i, j}$ are measurement matrix and zero-mean white Gaussian measurement noise with covariance matrix $R_{k}^{i, j}$, respectively. $y_{k}^{i, j}$ corresponds to the $j$ th measurement of sensor during time interval $k$. The set of measurements obtained by sensor $i$ during time interval is denoted by $Z_{k}^{i}$. Assume that $w(t), v_{k}^{i, j}$, and $x(0)$ are mutually independent. 
We also assume that the sensors in the network are time synchronized and time driven; therefore the sensors can easily obtain the time stamp through the digital communication network. The observations are exchanged between neighboring sensors once in every fusion interval. The packetdropping process at every communication links is modeled as independent identically distributed (i.i.d.) Bernoulli random process. We use Bernoulli random variable $\theta_{k}^{i, r}$ (for all $L^{i, r}=$ 1) to indicate whether a packet is successfully transmitted from sensor $i$ to sensor $r$ at $k$ th time instant. If $\theta_{k}^{i, r}=1$, the packet is successfully transmitted, otherwise, $\theta_{k}^{i, r}=0$ and the packet is dropped. Without loss of generality, it is assumed that $\theta_{k}^{i, i}=1$.

The $j$ th measurement sensor $r$ got from sensor $i$ during time interval $k$ can be written as

$$
\begin{gathered}
z_{k}^{i, j}=\theta_{k}^{i, r} y_{k}^{i, j}+\left(1-\theta_{k}^{i, r}\right) y_{k-1}^{i, j}, \\
i=1, \ldots, N, \quad j=1, \ldots, n_{k}^{i}, \quad \forall L^{i, r}=1,
\end{gathered}
$$

where $\lambda \stackrel{\Delta}{=} E\left\{\theta_{k}^{i, r}\right\}=\operatorname{Prob}\left\{\theta_{k}^{i, r}=1\right\}$ is the packet arriving probability (PAP) and $1-\lambda \triangleq 1-E\left\{\theta_{k}^{i, r}\right\}=\operatorname{Prob}\left\{\theta_{k}^{i, r}=0\right\}$ is the packet loss probability (PLP) [17].

\section{Distributed Asynchronous Fusion Algorithm}

3.1. Local Asynchronous Estimator. Without loss of generality, it is assumed that sensors in $\mathcal{N}_{i}$ totally obtain a set of $N_{k}^{i}$ asynchronous measurements during the $k$ th time interval. Measurement $z_{k}^{l, m}$ is provided by sensor $l\left(l \in \mathcal{N}_{i}\right)$ at time instant $\bar{t}_{k}^{l, m}\left(\bar{t}_{k}^{l, m} \in\left(t_{k-1}, t_{k}\right]\right)$. After sensor $i$ collects all $N_{k}^{i}$ asynchronous measurements which are provided by sensors in $\mathcal{N}_{i}$, it reorders these measurements in time sequence. Suppose the $j$ th measurement at sensor during the $k$ th time interval which is denoted by $Y_{k}^{i, j}$ corresponds to $z_{k}^{l, m}$, then we have

$$
Y_{k}^{i, j}=z_{k}^{l, m}=\theta_{k}^{l, i} y_{k}^{l, m}+\left(1-\theta_{k}^{l, i}\right) y_{k-1}^{l, m} .
$$

As mentioned before, we can obtain the time stamp of current measurement easily. The packet is successfully transmitted, if the time stamp of the latest measurement is in the present fusion interval, that is, $\theta_{k}^{l, i}=1$, otherwise $\theta_{k}^{l, i}=0$. We can simplify the measurement $Y_{k}^{i, j}$ as

$$
\begin{aligned}
Y_{k}^{i, j} & =\theta_{k}^{l, i} y_{k}^{l, m} \\
& =\theta_{k}^{l, i} h_{k}^{l, m} x\left(\bar{t}_{k}^{l, m}\right)+\theta_{k}^{l, i} v_{k}^{l, m} \\
& \triangleq \bar{\theta}_{k}^{i, j} \bar{H}_{k}^{i, j} x\left(t_{k}^{i, j}\right)+\bar{\theta}_{k}^{i, j} \bar{v}_{k}^{i, j},
\end{aligned}
$$

where $\bar{H}_{k}^{i, j}=h_{k}^{l, m}, t_{k}^{i, j}=\bar{t}_{k}^{l, m}, \bar{v}_{k}^{i, j}=v_{k}^{l, m}$, and $\bar{\theta}_{k}^{i, j}=\theta_{k}^{l, i}$.
The state $x\left(t_{k}^{i, j}\right)$ can be written by using (2) as

$$
x\left(t_{k}^{i, j}\right)=\Phi^{-1}\left(t_{k}, t_{k}^{i, j}\right) x\left(t_{k}\right)-\Phi^{-1}\left(t_{k}, t_{k}^{i, j}\right) w\left(t_{k}, t_{k}^{i, j}\right),
$$

where $\Phi^{-1}\left(t_{k}, t_{k}^{i, j}\right)=\Phi\left(t_{k}^{i, j}, t_{k}\right)$ is the backward state transition matrix.

By using (6) and (7), $Y_{k}^{i, j}$ can be rewritten as

$$
\begin{aligned}
Y_{k}^{i, j}= & \bar{H}_{k}^{i, j} x\left(t_{k}^{i, j}\right)+\bar{v}_{k}^{i, j} \\
= & \bar{\theta}_{k}^{i, j} \bar{H}_{k}^{i, j} \Phi^{-1}\left(t_{k}, t_{k}^{i, j}\right) x\left(t_{k}\right) \\
& -\bar{\theta}_{k}^{i, j} \bar{H}_{k}^{i, j} \Phi^{-1}\left(t_{k}, t_{k}^{i, j}\right) w\left(t_{k}, t_{k}^{i, j}\right)+\bar{\theta}_{k}^{i, j} \bar{v}_{k}^{i, j} \\
= & \xi_{k}^{i, j} H_{k}^{i, j} x\left(t_{k}\right)+\xi_{k}^{i, j} \Lambda_{k}^{i, j},
\end{aligned}
$$

where $H_{k}^{i, j}=\bar{H}_{k}^{i, j} \Phi^{-1}\left(t_{k}, t_{k}^{i, j}\right), \Lambda_{k}^{i, j}=\bar{v}_{k}^{i, j}-H_{k}^{i, j} w\left(t_{k}, t_{k-1}\right)$, and $\xi_{k}^{i, j}=\bar{\theta}_{k}^{i, j}$.

By denoting

$$
\begin{aligned}
& \mathscr{Y}_{k}^{i}=\left[\left(Y_{k}^{i, 1}\right)^{T}\left(Y_{k}^{i, 2}\right)^{T}, \ldots,\left(Y_{k}^{i, N_{k}^{i}}\right)^{T}\right]^{T}, \\
& \mathscr{H}_{k}^{i}=\left[\left(H_{k}^{i, 1}\right)^{T}\left(H_{k}^{i, 2}\right)^{T}, \ldots,\left(H_{k}^{i, N_{k}^{i}}\right)^{T}\right], \\
& \mathscr{L}_{k}^{i}=\left[\left(\Lambda_{k}^{i, 1}\right)^{T}\left(\Lambda_{k}^{i, 2}\right)^{T}, \ldots,\left(\Lambda_{k}^{i, N_{k}^{i}}\right)^{T}\right], \\
& \Xi_{k}^{i}=\operatorname{diag}\left\{\xi_{k}^{i, 1} I_{k}^{i, 1}, \xi_{k}^{i, 2} I_{k}^{i, 2}, \ldots, \xi_{k}^{i, N_{k}^{i}} I_{k}^{i, N_{k}^{i}}\right\},
\end{aligned}
$$

where $I_{k}^{i, j}, j=1,2, \ldots N_{k}^{i}$, are identity matrices with proper dimensions.

We can get an augmented measurement equation at sensor $i$ as

$$
\mathscr{Y}_{k}^{i}=\Xi_{k}^{i} \mathscr{H}_{k}^{i} x\left(t_{k}\right)+\Xi_{k}^{i} \mathscr{L}_{k}^{i}
$$

From (2), (3), (6), and (8) we have

$$
\begin{aligned}
& E\left[\xi_{k}^{i, j} \Lambda_{k}^{i, j} \mid \xi_{k}^{i, j}\right]= E\left[\bar{v}_{k}^{i, j}-H_{k}^{i, j} w\left(t_{k}, t_{k-1}\right) \mid \xi_{k}^{i, j}\right] \\
&= E\left[v_{k}^{l, m} \mid \xi_{k}^{i, j}\right]-H_{k}^{i, j} E\left[w\left(t_{k}, t_{k-1}\right) \mid \xi_{k}^{i, j}\right] \\
&= 0, \\
& E\left[\xi_{k}^{i, j} \Lambda_{k}^{i, j}\left(\xi_{k}^{i, j} \Lambda_{k}^{i, j}\right)^{T} \mid \xi_{k}^{i, j}\right] \\
&=\xi_{k}^{i, j} E\left[\left(\bar{v}_{k}^{i, j}-H_{k}^{i, j} w\left(t_{k}, t_{k}^{i, j}\right)\right)\right. \\
&\left.\times\left(\bar{v}_{k}^{i, j}-H_{k}^{i, j} w\left(t_{k}, t_{k}^{i, j}\right)\right)^{T}\right]
\end{aligned}
$$




$$
\begin{gathered}
=\xi_{k}^{i, j} E\left[\left(\bar{v}_{k}^{i, j}\right)\left(\bar{v}_{k}^{i, j}\right)^{T}\right] \\
+\xi_{k}^{i, j} E\left[\left(H_{k}^{i, j} w\left(t_{k}, t_{k}^{i, j}\right)\right)\right. \\
\left.\times\left(H_{k}^{i, j} w\left(t_{k}, t_{k}^{i, j}\right)\right)^{T}\right] \\
=\xi_{k}^{i, j} R_{k}^{i, j}+\xi_{k}^{i, j} H_{k}^{i, j} Q\left(t_{k}, t_{k}^{i, j}\right)\left(H_{k}^{i, j}\right)^{T} .
\end{gathered}
$$

$$
\begin{aligned}
& \text { Suppose } t_{k}^{i, j}>t_{k}^{i, l} \text {; then } \\
& \begin{array}{l}
E\left[\xi_{k}^{i, j} \Lambda_{k}^{i, j}\left(\xi_{k}^{i, l} \Lambda_{k}^{i, l}\right)^{T} \mid \xi_{k}^{i, j}, \xi_{k}^{i, l}\right] \\
=\xi_{k}^{i, j} E\left[\left(\bar{v}_{k}^{i, j}-H_{k}^{i, j} w\left(t_{k}, t_{k}^{i, j}\right)\right)\left(\bar{v}_{k}^{i, l}-H_{k}^{i, l} w\left(t_{k}, t_{k}^{i, l}\right)\right)^{T}\right] \\
=\xi_{k}^{i, j} E\left[\left(H_{k}^{i, j} w\left(t_{k}, t_{k}^{i, j}\right)\right)\left(H_{k}^{i, l} w\left(t_{k}, t_{k}^{i, l}\right)\right)^{T}\right] \\
=\xi_{k}^{i, j} \xi_{k}^{i, l} H_{k}^{i, j} Q\left(t_{k}, t_{k}^{i, j}\right)\left(H_{k}^{i, l}\right)^{T} .
\end{array}
\end{aligned}
$$

Thus, the augmented measurement noise $\mathscr{L}_{k}^{i}$ is zero-mean white Gaussian noise with covariance:

$$
\begin{aligned}
R_{k}^{i} & =\left[\begin{array}{ccc}
\xi_{k}^{i, 1}\left(R_{k}^{i, 1}+H_{k}^{i, 1} Q\left(t_{k}, t_{k}^{i, 1}\right) H_{k}^{i, 1}\right) & \ldots & \xi_{k}^{i, 1} \xi_{k}^{i, N_{k}^{i}} H_{k}^{i, 1} Q\left(t_{k}, t_{k}^{i, N_{k}^{i}}\right) H_{k}^{i, N_{k}^{i}} \\
\vdots & \ddots & \vdots \\
\xi_{k}^{i, N_{k}^{i}} \xi_{k}^{i, 1} H_{k}^{i, N_{k}^{i}} Q\left(t_{k}, t_{k}^{i, N_{k}^{i}}\right) H_{k}^{i, 1} & \ldots & \xi_{k}^{i, N_{k}^{i}}\left(R_{k}^{i, N_{k}^{i}}+H_{k}^{i, N_{k}^{i}} Q\left(t_{k}, t_{k}^{i, N_{k}^{i}}\right) H_{k}^{i, N_{k}^{i}}\right)
\end{array}\right] \\
& =\Xi_{k}^{i} \mathscr{R}_{k}^{i} \Xi_{k}^{i},
\end{aligned}
$$

where

$$
\mathscr{R}_{k}^{i}=\left[\begin{array}{ccc}
R_{k}^{i, 1}+H_{k}^{i, 1} Q\left(t_{k}, t_{k}^{i, 1}\right) H_{k}^{i, 1} & \ldots & H_{k}^{i, 1} Q\left(t_{k}, t_{k}^{i, N_{k}^{i}}\right) H_{k}^{i, N_{k}^{i}} \\
\vdots & \ddots & \vdots \\
H_{k}^{i, N_{k}^{i}} Q\left(t_{k}, t_{k}^{i, N_{k}^{i}}\right) H_{k}^{i, 1} & \ldots & R_{k}^{i, N_{k}^{i}}+H_{k}^{i, N_{k}^{i}} Q\left(t_{k}, t_{k}^{i, N_{k}^{i}}\right) H_{k}^{i, N_{k}^{i}}
\end{array}\right] .
$$

From (2), (8), and (11), we have

$$
\begin{aligned}
& E\left[w\left(t_{k}, t_{k-1}\right)\left(\xi_{k}^{i, j} \Lambda_{k}^{i, j}\right)^{T} \mid \xi_{k}^{i, j}\right] \\
& =\xi_{k}^{i, j} E\left[w\left(t_{k}, t_{k-1}\right)\left(\bar{v}_{k}^{i, j}-H_{k}^{i, j} w\left(t_{k}, t_{k}^{i, j}\right)\right)^{T}\right] \\
& =-\xi_{k}^{i, j} E\left[w\left(t_{k}, t_{k-1}\right)\left(H_{k}^{i, j} w\left(t_{k}, t_{k}^{i, j}\right)\right)^{T}\right] \\
& =-\xi_{k}^{i, j} Q\left(t_{k}, t_{k}^{i, j}\right)\left(H_{k}^{i, j}\right)^{T} .
\end{aligned}
$$

Further we have

$$
\begin{aligned}
& E\left[w\left(t_{k}, t_{k-1}\right)\left(\Xi_{k}^{i} \mathscr{L}_{k}^{i}\right)^{T}\right] \\
& =\left[E\left[w\left(t_{k}, t_{k-1}\right)\left(\xi_{k}^{i, j} \Lambda_{k}^{i, 1}\right)^{T}\right] E\left[w\left(t_{k}, t_{k-1}\right)\left(\xi_{k}^{i, j} \Lambda_{k}^{i, 2}\right)^{T}\right]\right. \\
& \left.\quad \ldots E\left[w\left(t_{k}, t_{k-1}\right)\left(\xi_{k}^{i, j} \Lambda_{k}^{i, N_{k}^{i}}\right)^{T}\right]\right]
\end{aligned}
$$

$$
\begin{aligned}
& =\Xi_{k}^{i} \mathscr{V}_{k}^{i} \\
& =V_{k}^{i},
\end{aligned}
$$

where $\mathscr{V}_{k}^{i}=\left[E\left[w\left(t_{k}, t_{k-1}\right)\left(\Lambda_{k}^{i, 1}\right)^{T}\right] E\left[w\left(t_{k}, t_{k-1}\right)\left(\Lambda_{k}^{i, 2}\right)^{T}\right] \ldots\right.$ $\left.E\left[w\left(t_{k}, t_{k-1}\right)\left(\Lambda_{k}^{i, N_{k}^{i}}\right)^{T}\right]\right]$.

It can be observed from (19) that the discrete-time process noise and the augmented measurement noise are cross correlated; this correlation comes from the common continuous-time process noise [40].

For sensor $i$, the asynchronous local estimation problem in $\mathcal{N}_{i}$ is converted to an estimation problem of the following system:

$$
\begin{gathered}
x\left(t_{k}\right)=\Phi\left(t_{k}, t_{k-1}\right) x\left(t_{k-1}\right)+w\left(t_{k}, t_{k-1}\right), \\
Y_{k}^{i}=H_{k}^{i} x\left(t_{k}\right)+\Lambda_{k}^{i},
\end{gathered}
$$

where $Y_{k}^{i}=\mathscr{Y}_{k}^{i}, H_{k}^{i}=\Xi_{k}^{i} \mathscr{H}_{k}^{i}$, and $\Lambda_{k}^{i}=\Xi_{k}^{i} \mathscr{L}_{k}^{i}$. 
Thus a local asynchronous estimation method is summarized in Theorem 1 in an optimal batch asynchronous data fusion fashion [38].

Theorem 1 (local asynchronous estimator). For sensor $i$, after it gets asynchronous measurements from sensors in $N_{i}$, the local asynchronous estimator at sensor $i$ is summarized as follow: given the local state estimate $\hat{x}_{k-1}^{i}$ of system (1) and its error covariance matrix $M_{k-1}^{i}$ at time $t_{k-1}$, the local estimate and its error covariance matrix at time $t_{k}$ are given by

$$
\begin{gathered}
\bar{x}_{k}^{i}=\Phi\left(t_{k}, t_{k-1}\right) \hat{x}_{k-1}^{i}, \\
\bar{M}_{k}^{i}=\Phi\left(t_{k}, t_{k-1}\right) M_{k-1}^{i} \Phi\left(t_{k}, t_{k-1}\right)+Q\left(t_{k}, t_{k-1}\right), \\
M_{k}^{i}=\left\{\left(\bar{M}_{k}^{i}\right)^{-1}+\left[H_{k}^{i}+\left(V_{k}^{i}\right)^{T}\left(\bar{M}_{k}^{i}\right)^{-1}\right]^{T}\right. \\
\times\left[R_{k}^{i}-\left(V_{k}^{i}\right)^{T}\left(\bar{M}_{k}^{i}\right)^{-1} V_{k}^{i}\right]^{-1} \\
\left.\times\left[H_{k}^{i}+\left(V_{k}^{i}\right)^{T}\left(\bar{M}_{k}^{i}\right)^{-1}\right]\right\}^{-1}, \\
\widehat{x}_{k}^{i}=\bar{x}_{k}^{i}+M_{k}^{i}\left[H_{k}^{i}+\left(V_{k}^{i}\right)^{T}\left(\bar{M}_{k}^{i}\right)^{-1}\right]^{T} \\
\times\left[R_{k}^{i}-\left(V_{k}^{i}\right)^{T}\left(\bar{M}_{k}^{i}\right)^{-1} V_{k}^{i}\right]^{-1} \\
\times\left(Y_{k}^{i}-H_{k}^{i} \bar{x}_{k}^{i}\right),
\end{gathered}
$$

where $\hat{x}_{k}^{i}$ and $\bar{x}_{k+1}^{i}$ are the state estimate and one-step predicted estimate, respectively, $M_{k}^{i}$ and $\bar{M}_{k}^{i}$ are the covariance matrices of filtering error and one-step prediction error, respectively, and the initial values of $\bar{x}_{k+1}^{i}$ and $\bar{M}_{k}^{i}$ are given, respectively, by $x_{0}$ and $M_{0}$, where $x_{0}$ is a normally distributed random variable with mean equal to the initial truth ground of the state $x$ and covariance $M_{0}$.

Proof. For neighborhood $\mathcal{N}_{i}$, the local asynchronous estimation problem at sensor $i$ is converted to the state estimation problem of system (20). This is a classical filtering problem with noise cross-covariance which can be solved by using the Kalman filter [40] for correlated process and measurement noises.

Remark 2. The random packets losses have been taken into account in Theorem 1 . And in Theorem 1 each sensor in the sensor network generates a local estimate at the fusion time instant by using measurements only from its neighbors. These local estimates are suboptimal in the sense that not all the measurements in the sensor network are used. Therefore, a fusion criterion, which will improve each local estimation performance, weighted by matrices will be developed later in this paper. The main results will be presented in the following subsection.

3.2. CI-Based Fusion Estimator. In this subsection, a fusion criterion weighted by matrices is developed to generate fusion estimate at every sensor, and it takes into consideration the random packets dropouts. The criterion is first given in the following lemma in [16].

Lemma 3. Let $\widehat{\eta}_{1} ; \widehat{\eta}_{1}, \ldots, \widehat{\eta}_{n}$ denote unbiased estimates for the unknown state vector $\eta$ and let $P_{1}, P_{2}, \ldots, P_{n}$ denote the corresponding estimated covariance matrices; the CI fusion estimator is given by the following convex combination:

$$
\begin{gathered}
P_{0}^{-1}=\sum_{n=1}^{N} \omega_{n} P_{n}^{-1}, \\
P_{0}^{-1} \eta_{0}=\sum_{n=1}^{N} \omega_{n} P_{n}^{-1} \eta_{n},
\end{gathered}
$$

where $0 \leq \omega_{n} \leq 1$ and $\omega_{1}+\omega_{2}+\cdots+\omega_{n}=1$ hold .

When local estimates calculated by the local estimators in Theorem 1 are available at the sensors in the sensor network, sensor $i$ collects them from its neighborhood $\mathcal{N}_{i}$ to generate a fused estimate according to the fusion rule based on covariance intersection. For the packet-dropping process, it is also modeled as i.i.d. Bernoulli random process. Denote $\mu_{k}^{i, r}$ (for all $L^{i, r}=1$ ) as the packet transmit indicator. It is also assumed that $\mu_{k}^{i, i}=1$.

Theorem 4 (CI-based fusion estimator). By gathering the local estimates provided by the sensors in neighborhood $N_{i}$, the distributed weighted fusion filter at sensor $i$ is given by

$$
x_{k}^{i}=\sum_{l \in N_{i}} S_{k}^{l} \hat{x}_{k}^{l}
$$

where

$$
\begin{gathered}
S_{k}^{l}=\mu_{k}^{i, l} \alpha_{k}^{l}\left(\Psi_{k}^{i}\right)^{-1}\left(M_{k}^{l}\right)^{-1}, \\
\Psi_{k}^{i}=\sum_{l \in \mathcal{N}_{i}} \mu_{k}^{i, l} \alpha_{k}^{l}\left(M_{k}^{l}\right)^{-1} .
\end{gathered}
$$

$\alpha_{k}^{l}$ are subject to $0 \leq \alpha_{k}^{l} \leq 1, \sum_{l \in \mathcal{N}_{i}} \mu_{k}^{i, l} \alpha_{k}^{l}=1$.

Proof. Theorem 4 follows directly from Lemma 3.

Remark 5. Different choices of $\alpha_{k}^{l}$ can be used to optimize the update with respect to different performance criteria, such as minimizing the trace or determinant of $\Psi_{k}^{i}$. However such optimization is nonlinear and a high computation load is required for computing the optimal $\alpha_{k}^{l}$ [41]. Thus, several fast CI algorithms that produce suboptimal solutions have been proposed in terms of trace or determinant minimization [41, 42]. In what follows, we choose to minimize the trace of $\Psi_{k}^{i}$. For sensor $i$, a suboptimal noniterative weighting coefficients solution for fast CI fusion is given by

$$
\alpha_{k}^{l}=\frac{1 / \operatorname{tr}\left(M_{k}^{l}\right)}{\sum_{j \in \mathcal{N}_{i}} \mu_{k}^{i, j}\left(1 / \operatorname{tr}\left(M_{k}^{j}\right)\right)}, \quad l \in \mathcal{N}_{i} .
$$

For clarity, the asynchronous distributed fusion algorithm is summarized in Algorithm 1. 

(1) Initialization: $\bar{M}_{k}^{i}=M_{0}, \bar{x}_{k+1}^{i}=x_{0}$.
(2) Obtain local measurements $z_{k}^{i, p}$.
(3) Broadcast message $m_{k}^{1, i}=\left\{z_{k}^{i, p}\right\}\left(p=1,2, \ldots, n_{k}^{i}\right)$ to neighbors.
(4) Receive messages $m_{k}^{1, l}, l \in \mathcal{N}_{i}$, from neighbors.
(5) Compute $Y_{k}^{i}, H_{k}^{i}, R_{k}^{i}$, and $V_{k}^{i}$ by using (9)-(19).
(6) Calculate the local estimate $\widehat{x}_{k}^{i}$ by using Theorem 1.
(7) Broadcast message $m_{k}^{2, i}=\left\{M_{k}^{i}, \widehat{x}_{k}^{i}\right\}$ to neighbors.
(8) Receive messages $m_{k}^{2, l}, l \in \mathcal{N}_{i}$, from neighbors.
(9) Calculate the fusion estimate $x_{k}^{i}$ by using Theorem 4 and (25).

Algorithm 1

Remark 6. In the distributed asynchronous fusion filter presented in Algorithm 1, each sensor in sensor network broadcasts its local measurement information and local estimate to its neighboring sensors; for every sensor, more information from different sensors, not only the neighboring ones, will be utilized to generate the fusion estimates, which helps improve the local estimate performance and reduce the disagreement of local estimates. Further, not all target information in the sensor network is used to generate the fusion estimate at one sensor; meanwhile the method we used to compute coefficient $\alpha_{k}^{l}$ is suboptimal; therefore the fusion estimate is a suboptimal estimate, but it is an improved one compared with the local one.

\section{Simulation}

In this section, the effectiveness of the proposed estimator design method is demonstrated by presenting simulations of a target tracking system. Consider the following continuoustime dynamic system:

$$
\dot{x}(t)=\left[\begin{array}{ll}
0 & 1 \\
0 & 0
\end{array}\right] x(t)+\left[\begin{array}{l}
0 \\
1
\end{array}\right] w(t)
$$

where $x(t) \in \mathscr{R}^{2}$ and $w(t)$ is zero-mean white Gaussian noise with variance $q=1$; by discretizing the continuous-time linear system (26), we have

$$
x\left(t_{k}\right)=\left[\begin{array}{cc}
1 & t_{k}-t_{k-1} \\
0 & 1
\end{array}\right] x\left(t_{k-1}\right)+w\left(t_{k}, t_{k-1}\right),
$$

where the process noise $w\left(t_{k}\right)$ is a white Gaussian noise sequence with zero mean and covariance

$$
Q\left(t_{k}, t_{k-1}\right)=\left[\begin{array}{ll}
\frac{\left(t_{k}-t_{k-1}\right)^{3}}{3} & \frac{\left(t_{k}-t_{k-1}\right)^{2}}{2} \\
\frac{\left(t_{k}-t_{k-1}\right)^{2}}{2} & t_{k}-t_{k-1}
\end{array}\right]
$$

The state of system (27) is observed by a sensor network with 12 sensor nodes, and the topology of the sensor network is shown in Figure 3. The observation equations of the sensors are given by (3) with measurement matrices $h_{k}^{i, j}=\left[\begin{array}{ll}1 & 0\end{array}\right]$,

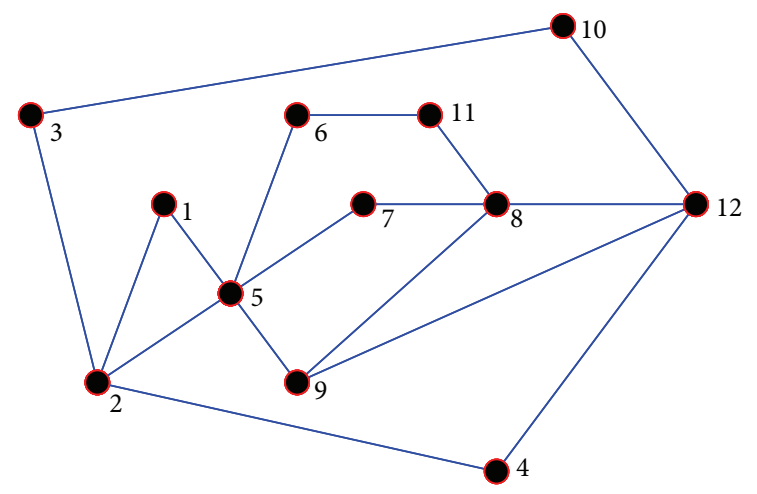

FIgURE 1: The network topology with 12 sensor nodes.

$i=1, \ldots, 12$ and $j=1, \ldots, n_{k}^{i}$; measurement noise variances $R_{k}^{i, j}=25, i=1, \cdots, 12$ and $j=1, \cdots, n_{k}^{i}$; and sampling periods $T_{1}=T_{2}=T_{3}=0.3 s, T_{4}=T_{5}=T_{6}=T_{7}=T_{8}=0.4 s$, and $T_{9}=T_{10}=T_{11}=T_{12}=0.6 \mathrm{~s}$, respectively. The fusion operation is performed with period $T_{F}=1 s$ (i.e., $t_{k}-t_{k-1}=1$ ) and initial time instant $t_{F}=1 s$.

For performance comparison, root mean square errors (RMSE) of position and velocity given by [40]

$$
\operatorname{RMSE}_{k}(i)=\sqrt{\frac{1}{M} \sum_{m=1}^{M}\left[x_{k}^{m, e}(i)-x_{k}^{m, \text { true }}(i)\right]^{2}}
$$

are used, where $M=100$ is the Monte-Carlo simulation times, $x_{k}^{m \text {,true }}(i)$ is the ground truth of the $i$ th component of the state vector in the $m$ th run, and $x_{k}^{m, e}(i)$ is the corresponding estimate; the disagreement potential of the estimates in the neighborhood $\mathcal{N}_{i}$ is defined as

$$
\Delta\left(t_{k}\right)=\frac{1}{2 \mathscr{D}_{i, j \in \mathcal{N}_{i}}} \sum_{i}\left\|x_{k}^{e, i}-x_{k}^{e, j}\right\|^{2}
$$

where $x_{k}^{e, i}$ is the estimate of sensor $i$ at time instant $t_{k}, P_{k}^{i}$ is the corresponding error covariance, and the simulation ends after 20 seconds.

We present the simulation results without packet losses firstly. As shown in Figure 1, sensors 2 and 12 are directly 

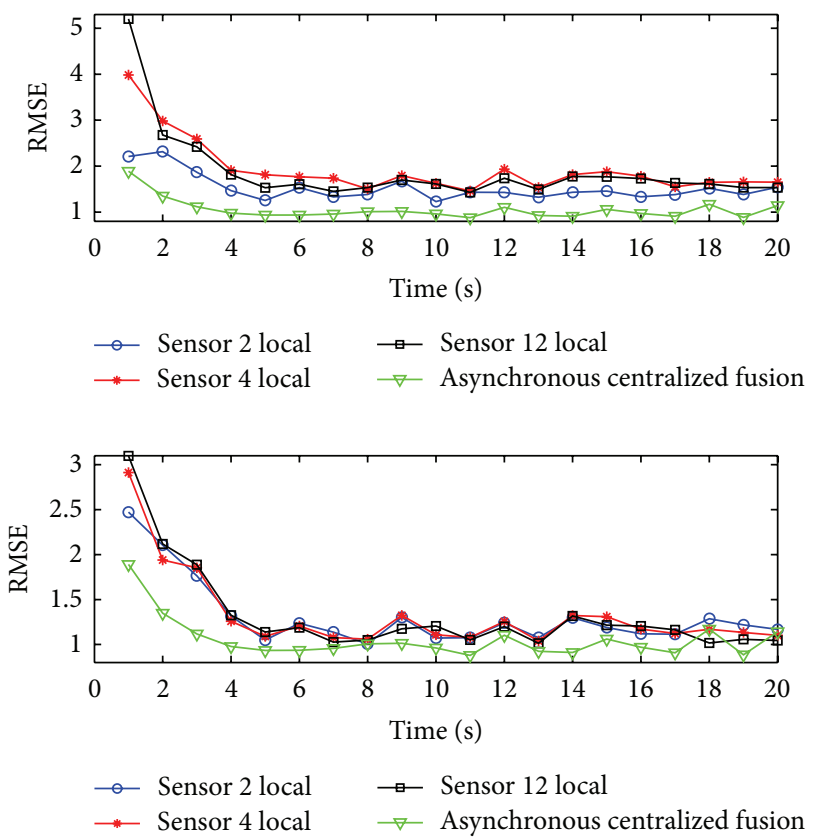

FIgURE 2: Performance comparison of local estimates, fusion estimates and centralized asynchronous fusion estimate.

connected to sensor 4 , thus neighborhood $\mathcal{N}_{4}$ consists of sensors 2, 4, and 12, with sampling periods $T_{2}=T_{4}=0.3 \mathrm{~s}$ and $T_{12}=0.6 s$, respectively. We take estimate performance of sensors in neighborhood $\mathcal{N}_{4}$ to testify the effectiveness of the proposed algorithm. At each fusion instant $t_{k}$, local estimate at sensor 4 is generated by collecting measurements from itself and sensors 2 and 12, and then sensor 4 collects local estimates from itself and sensors 2 and 12 to form fused estimates by using Theorem 4 and (25). Since trends of the simulation results are the same for both components, only the first component of the state vector is shown and analyzed below, and the simulation results of the proposed algorithm are compared with the optimal linear minimum meansquared error centralized asynchronous fusion algorithm in [22].

Figure 2 compares estimate performance of the proposed local asynchronous estimator and fusion estimator with the centralized asynchronous fusion estimator proposed in [22]. Although the performance of local estimator and fusion estimator is close to the centralized one, the centralized asynchronous fusion algorithm outperforms them both. This verifies our conclusion that the proposed local asynchronous estimator and fusion estimator are suboptimal as we stated in Remarks 2 and 6.

Figure 3 compares the performance of the proposed local asynchronous estimator and fusion estimator. It can be observed that the RMSE of fusion estimates is smaller than that of the corresponding local estimates; this verifies that the fusion estimates improve the performance of local estimates.

Figure 4 compares the disagreement of estimates obtained by the proposed local asynchronous estimator
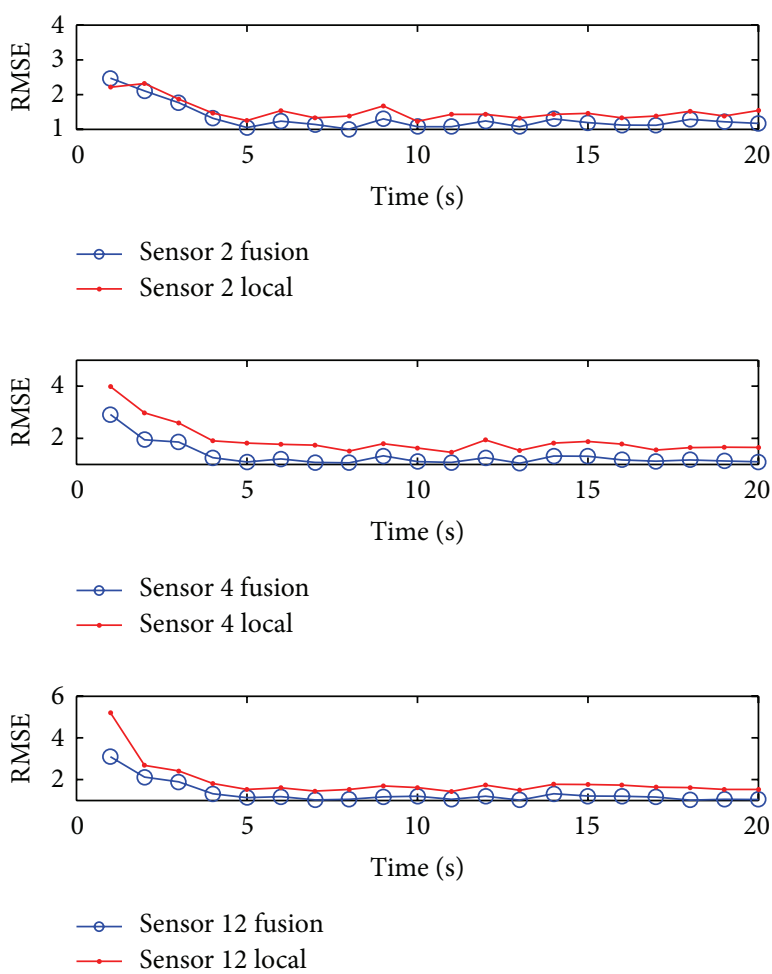

Figure 3: Performance comparison of local estimates and fusion estimates at sensors 2,4 , and 12 .

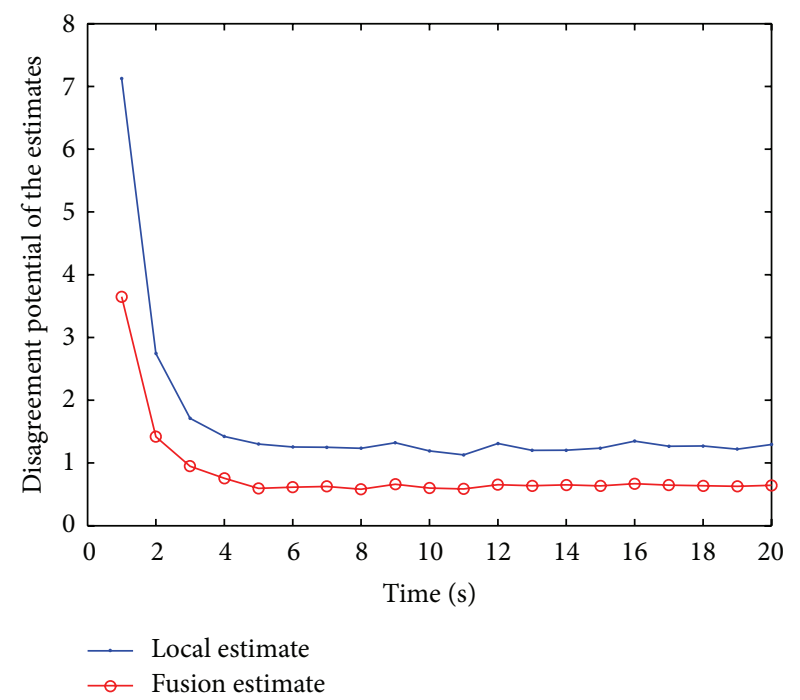

FIGURE 4: Disagreement potential comparison of the estimates (each curve is determined by averaging 100 random runs of Algorithm 1).

and fusion estimator; the fusion estimator gains smaller disagreement potential than the corresponding local one. This verifies our conclusion that fusion estimator reduces the disagreement among different sensors.

Figures 5 and 6 shows the filtering performances of the sensors in $N_{4}$ with different PLPs. It can be observed that 

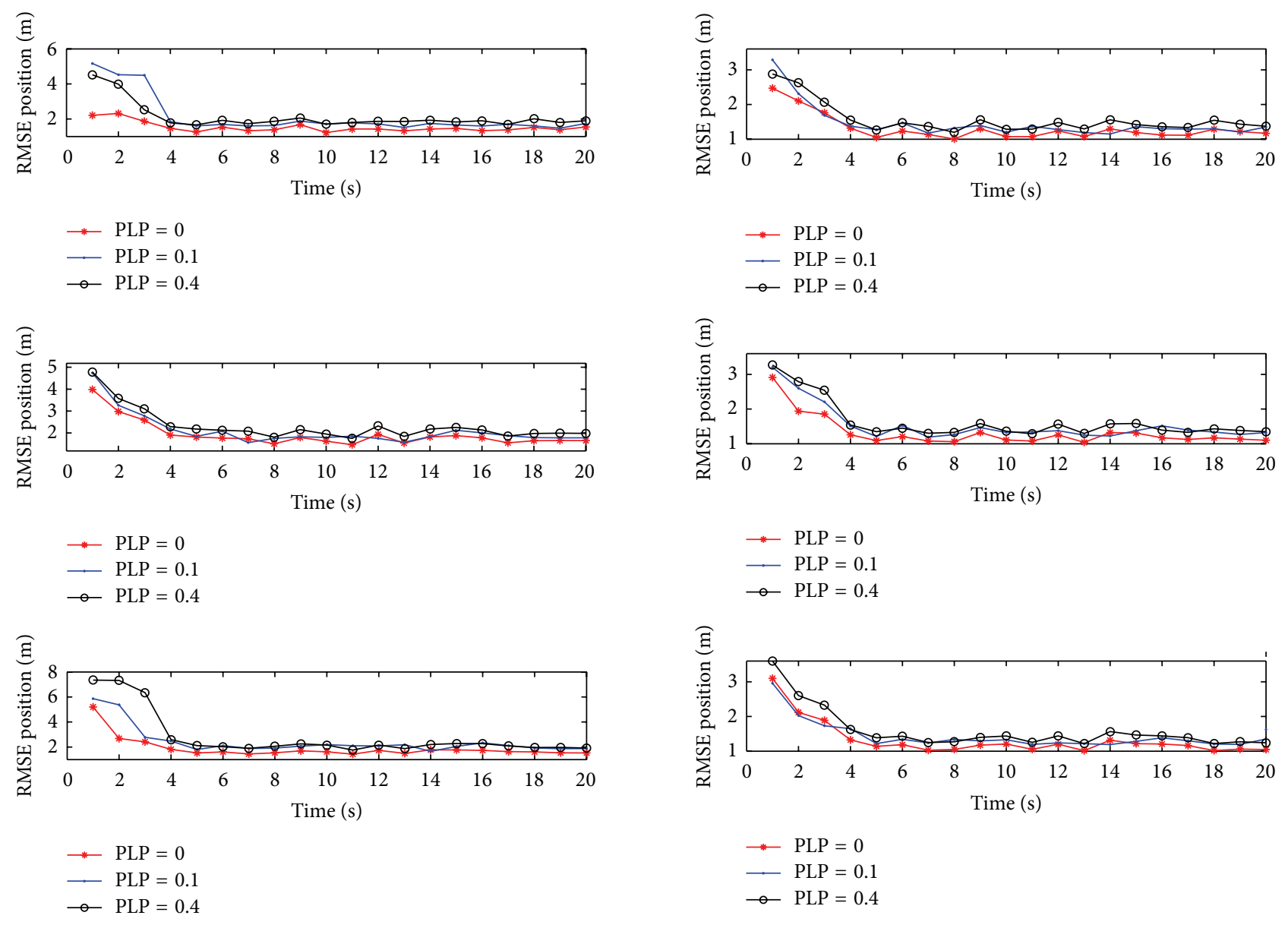

FIgURE 5: Local estimator performances with PLP $=0,0.1,0.4$.

packet dropout degrades estimation performance. The fusion estimator performance still outperforms local estimator and both estimators RMSEs converge. This demonstrates the effectiveness of the proposed distributed asynchronous fusion algorithm.

\section{Conclusion}

A CI-based distributed asynchronous fusion algorithm has been proposed for decentralized asynchronous sensor networks with random packet dropouts in this paper. The proposed distributed asynchronous fusion algorithm is applicable to decentralized asynchronous sensor networks; it is capable of improving estimates and reducing disagreement in comparison with the local ones; meanwhile it is also able to deal with random packet losses. The simulation results demonstrate the effectiveness of the proposed distributed asynchronous fusion algorithm.

\section{Conflict of Interests}

The authors declare that there is no conflict of interests regarding the publication of this paper.

\section{Acknowledgments}

This work was supported by the National Natural Science Foundation of China (no. 61104186 and no. 61273076) and the Natural Science Foundation of Jiangsu Province (no. BK2012801).

\section{References}

[1] C. Chong, S. Mori, and K. Chang, "Adaptive distributed estimation," in Proceedings of the IEEE Conference on Decision Control,, vol. 26, pp. 2233-2238, 1987.

[2] K. C. Chang, R. K. Saha, and Y. Bar-Shalom, "On optimal trackto-track fusion," IEEE Transactions on Aerospace and Electronic Systems, vol. 33, no. 4, pp. 1271-1276, 1997.

[3] X. Li, K. Zhang, J. Zhao, and Y. Zhu, "Optimal linear estimation fusion-part V: relationships," in Proceedings of the 5th International Conference on Information Fusion, pp. 497-504, 2002.

[4] S. Mori, W. H. Barker, C.-Y. Chong, and K.-C. Chang, "Track association and track fusion with nondeterministic target dynamics," IEEE Transactions on Aerospace and Electronic Systems, vol. 38, no. 2, pp. 659-668, 2002.

[5] Y. Zhu, E. Song, J. Zhou, and Z. You, "Optimal dimensionality reduction of sensor data in multisensor estimation fusion," IEEE Transactions on Signal Processing, vol. 53, no. 5, pp. 1631-1639, 2005. 
[6] S. Marano, V. Matta, and P. Willett, "Distributed estimation in large wireless sensor networks via a locally optimum approach," IEEE Transactions on Signal Processing, vol. 56, no. 2, pp. 748756, 2008.

[7] I. D. Schizas, G. B. Giannakis, and Z.-Q. Luo, "Distributed estimation using reduced-dimensionality sensor observations," IEEE Transactions on Signal Processing, vol. 55, no. 8, pp. 42844299, 2007.

[8] F. S. Cattivelli, C. G. Lopes, and A. H. Sayed, "Diffusion recursive least-squares for distributed estimation over adaptive networks," IEEE Transactions on Signal Processing, vol. 56, no. 5, pp. 1865-1877, 2008.

[9] R. Carli, A. Chiuso, L. Schenato, and S. Zampieri, "Distributed Kalman filtering based on consensus strategies," IEEE Journal on Selected Areas in Communications, vol. 26, no. 4, pp. 622633, 2008.

[10] S. Kar and J. M. F. Moura, "Gossip and distributed Kalman filtering: weak consensus under weak detectability," IEEE Transactions on Signal Processing, vol. 59, no. 4, pp. 1766-1784, 2011.

[11] R. Olfati-Saber, "Distributed Kalman filtering for sensor networks," in Proceedings of the 46th IEEE Conference on Decision and Control (CDC '07), pp. 5492-5498, December 2007.

[12] R. Olfati-Saber, "Distributed Kalman filter with embedded consensus filters," in Proceedings of the 44th IEEE Conference on Decision and Control, and the European Control Conference (CDC-ECC '05), pp. 8179-8184, December 2005.

[13] F. S. Cattivelli and A. H. Sayed, "Diffusion strategies for distributed Kalman filtering and smoothing," IEEE Transactions on Automatic Control, vol. 55, no. 9, pp. 2069-2084, 2010.

[14] F. Cattivelli and A. H. Sayed, "Diffusion distributed Kalman filtering with adaptive weights," in Proceedings of the 43rd Asilomar Conference on Signals, Systems and Computers, pp. 908-912, November 2009.

[15] S. J. Julier and J. K. Uhlmann, "Non-divergent estimation algorithm in the presence of unknown correlations," in Proceedings of the American Control Conference, vol. 4, pp. 2369-2373, June 1997.

[16] S. Julier and J. Uhlmann, General Decentralized Data Fusion with Covariance Intersection, Handbook of Multisensor Data Fusion, 2001.

[17] J. Hu, L. Xie, and C. Zhang, "Diffusion Kalman filtering based on covariance intersection," IEEE Transactions on Signal Processing, vol. 60, no. 2, pp. 891-902, 2012.

[18] H. Dong, Z. Wang, and H. Gao, "Distributed $\mathrm{H}_{\infty}$ filtering for a class of Markovian jump nonlinear time-delay systems over lossy sensor networks," IEEE Transactions on Industrial Electronics, vol. 60, no. 10, pp. 4665-4672, 2013.

[19] L. Ma, Z. Wang, Y. Bo, and Z. Guo, "Robust $H_{\infty}$ sliding mode control for nonlinear stochastic systems with multiple data packet losses," International Journal of Robust and Nonlinear Control, vol. 22, no. 5, pp. 473-491, 2012.

[20] L. Ma, Z. Wang, Y. Bo, and Z. Guo, "Finite-horizon $H_{2} / H_{\infty}$ control for a class of nonlinear Markovian jump systems with probabilistic sensor failures," International Journal of Control, vol. 84, no. 11, pp. 1847-1857, 2011.

[21] D. Ding, Z. Wang, H. Dong, and H. Shu, "Distributed $H_{\infty}$ state estimation with stochastic parameters and nonlinearities through sensor networks: the finite-horizon case," Automatica, vol. 48, no. 8, pp. 1575-1585, 2012.

[22] H. Dong, Z. Wang, and H. Gao, "Distributed $H_{\infty}$ filtering for a class of Markovian jump nonlinear time-delay systems over lossy sensor networks," IEEE Transactions on Industrial Electronics, vol. 60, no. 10, pp. 4665-4672, 2013.

[23] H. Dong, Z. Wang, and H. Gao, "Distributed filtering for a class of time-varying systems over sensor networks with quantization errors and successive packet dropouts," IEEE Transactions on Signal Processing, vol. 60, no. 6, pp. 3164-3173, 2012.

[24] N. E. Nahi, "Optimal recursive estimation with uncertain observation," IEEE Transactions on Information Theory, vol. 15, no. 4, pp. 457-462, 1969.

[25] S. Kar, B. Sinopoli, and J. M. F. Moura, "Kalman filtering with intermittent observations: weak convergence to a stationary distribution," IEEE Transactions on Automatic Control, vol. 57, no. 2, pp. 405-420, 2012.

[26] S. Nakamori, R. Caballero-Águila, A. Hermoso-Carazo, and J. Linares-Pérez, "Linear recursive discrete-time estimators using covariance information under uncertain observations," Signal Processing, vol. 83, no. 7, pp. 1553-1559, 2003.

[27] Z. Wang, D. W. C. Ho, and X. Liu, "Robust filtering under randomly varying sensor delay with variance constraints," IEEE Transactions on Circuits and Systems, vol. 51, no. 6, pp. 320-326, 2004.

[28] L. Schenato, "Optimal estimation in networked control systems subject to random delay and packet loss," in Proceedings of the 45th IEEE Conference on Decision and Control (CDC '06), pp. 5615-5620, December 2006.

[29] B. Sinopoli, L. Schenato, M. Franceschetti, K. Poolla, M. I. Jordan, and S. S. Sastry, "Kalman filtering with intermittent observations," IEEE Transactions on Automatic Control, vol. 49, no. 9, pp. 1453-1464, 2004.

[30] M. Sahebsara, T. Chen, and S. L. Shah, "Optimal $H_{2}$ filtering with random sensor delay, multiple packet dropout and uncertain observations," International Journal of Control, vol. 80, no. 2, pp. 292-301, 2007.

[31] S. L. Sun, "Optimal linear estimation for networked systems with one-step random delays and multiple packet dropouts," Acta Automatica Sinica, vol. 38, no. 3, pp. 349-356, 2012.

[32] J. Ma and S. Sun, "Centralized fusion estimators for multisensor systems with random sensor delays, multiple packet dropouts and uncertain observations," IEEE Transactions on Signal Processing, vol. 13, no. 4, 2013.

[33] A. T. Alouani and T. R. Rice, "On optimal asynchronous track fusion," in Proceedings of the 1st Austalian Data Fusion Symposium, pp. 147-152, November 1996.

[34] A. T. Alouani and T. R. Rice, "Asynchronous fusion of correlated tracks," in Acquisition, Tracking and Pointing XII, Proceedings of SPIE, pp. 113-118, April 1998.

[35] G. A. Watson, T. R. Rice, and A. T. Alouani, "Optimal track fusion with feedback for multiple asynchronous measurements," in Aquisition, Tracking, and Pointing XIV, Proceedings of SPIE, pp. 20-33, April 2000.

[36] A. T. Alouani, J. E. Gray, and D. H. McCabe, "Theory of distributed estimation using multiple asynchronous sensors," IEEE Transactions on Aerospace and Electronic Systems, vol. 41, no. 2, pp. 717-722, 2005.

[37] L. P. Yan, B. S. Liu, and D. H. Zhou, "Asynchronous multirate multisensor information fusion algorithm," IEEE Transactions on Aerospace and Electronic Systems, vol. 43, no. 3, pp. 1135-1146, 2007.

[38] H. Yanyan, D. Zhansheng, and H. Chongzhao, "Optimal batch asynchronous fusion algorithm," in Proceedings of the IEEE International Conference on Vehicular Electronics and Safety, pp. 237-240, October 2005. 
[39] Y. Hu, Z. Duan, and D. Zhou, "Estimation fusion with general asynchronous multi-rate sensors," IEEE Transactions on Aerospace and Electronic Systems, vol. 46, no. 4, pp. 2090-2102, 2010.

[40] D. Simon, Optimal State Estimation: Kalman, $H_{\infty}$, and Nonlinear Approaches, Wiley-Interscience, Hoboken, NJ, USA, 2006.

[41] P. O. Arambel, C. Rago, and R. K. Mehra, "Covariance intersection algorithm for distributed spacecraft state estimation," in Proceedings of the American Control Conference, pp. 4398-4403, June 2001.

[42] Y. Wang and X. R. Li, "A fast and fault-tolerant convex combination fusion algorithm under unknown cross-correlation," in Proceedings of the 12th International Conference on Information Fusion (FUSION '09), pp. 571-578, July 2009. 


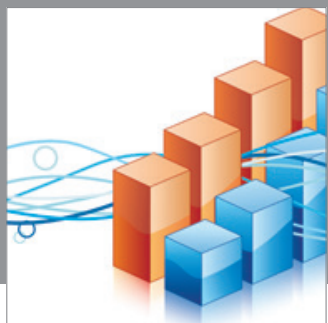

Advances in

Operations Research

mansans

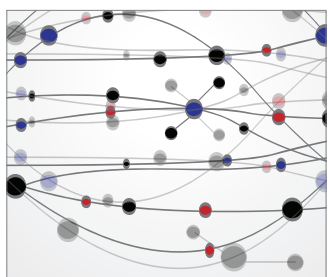

The Scientific World Journal
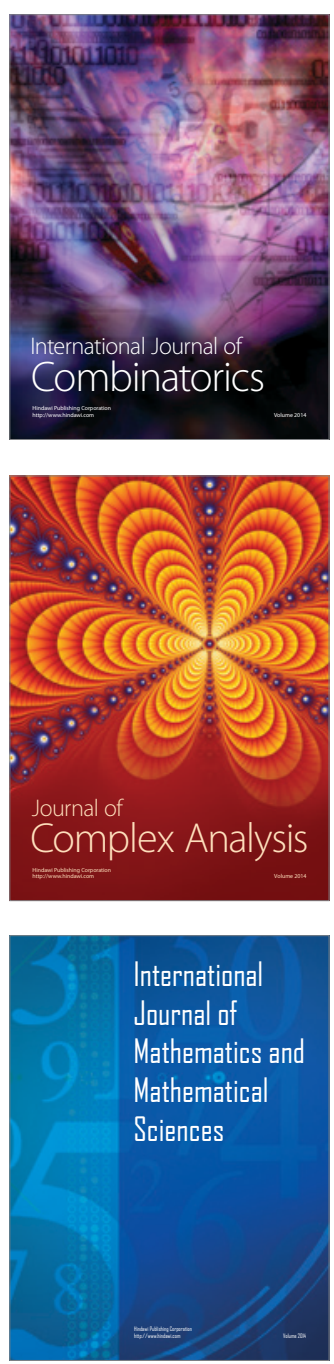
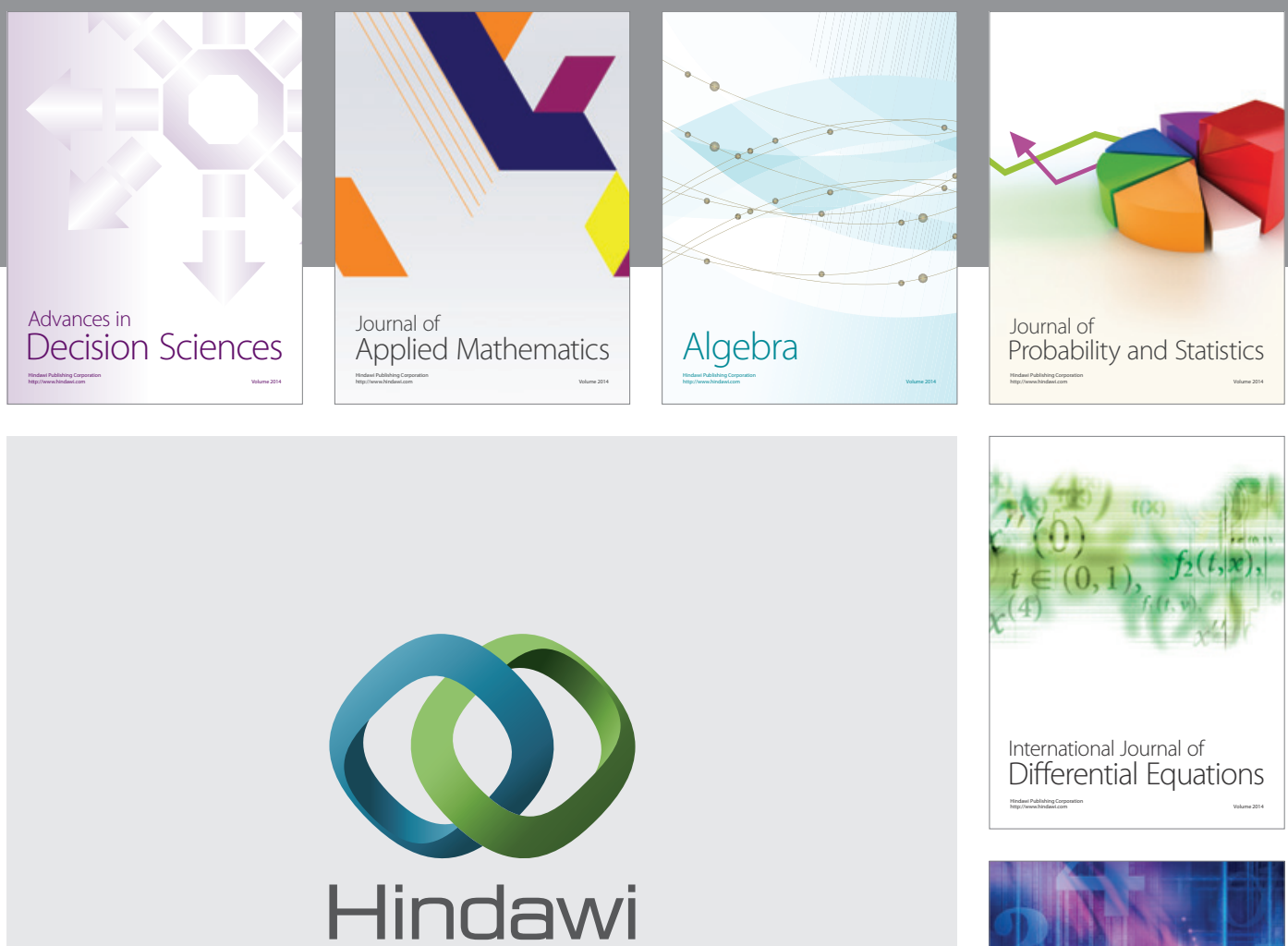

Submit your manuscripts at http://www.hindawi.com
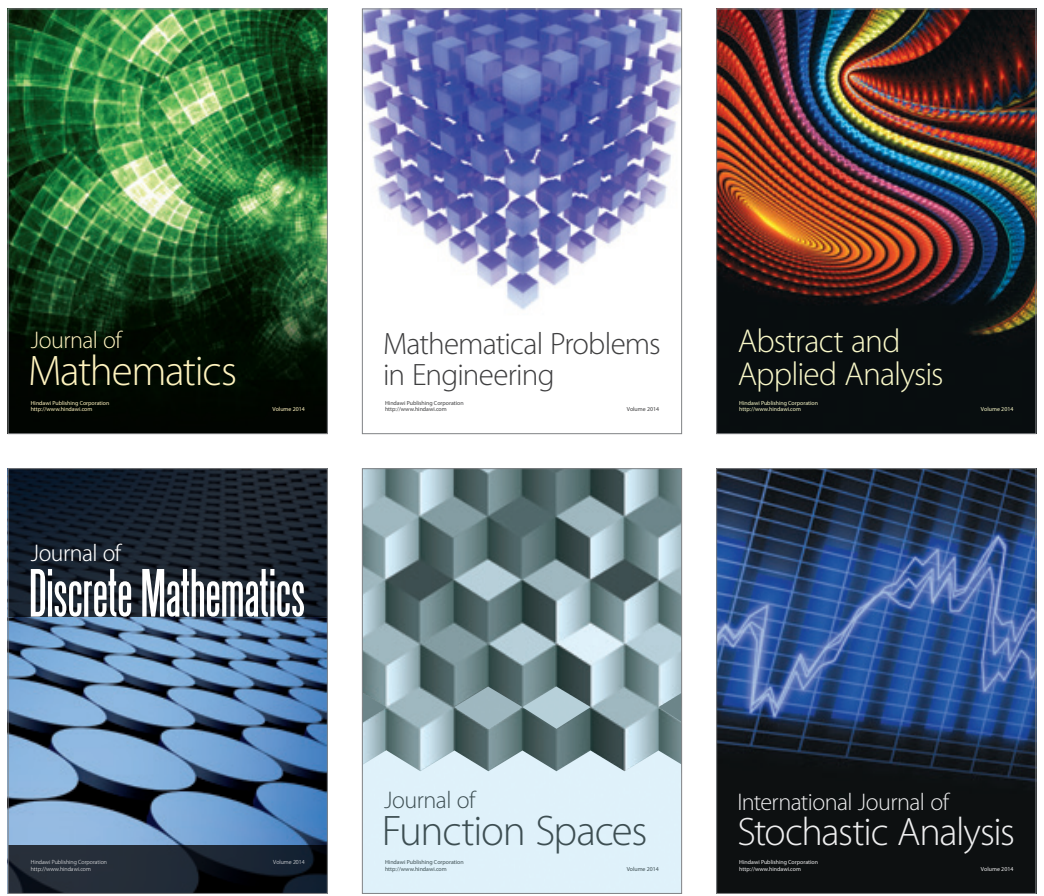

Journal of

Function Spaces

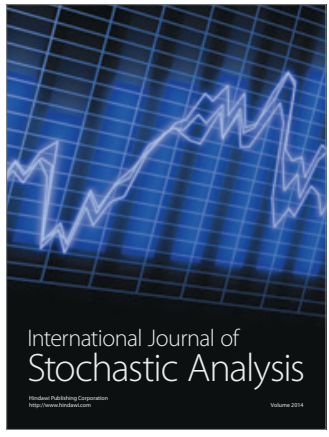

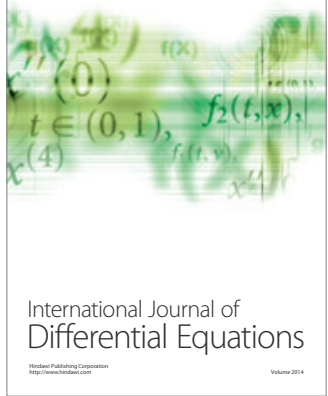
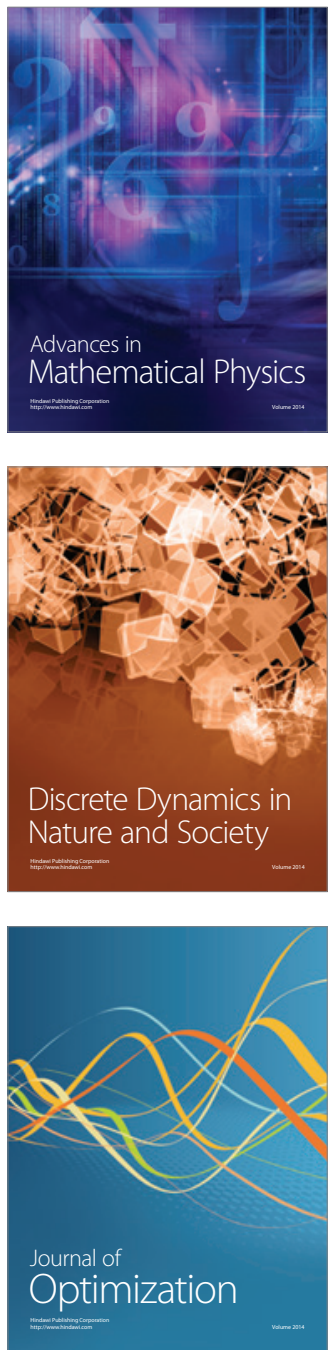